\title{
Prospective use of whole genome sequencing (WGS) detected a multi-country outbreak of Salmonella Enteritidis
}

\author{
T. INNS ${ }^{1,2,3 *}$, P. M. ASHTON ${ }^{4}$, S. HERRERA-LEON ${ }^{5}$, J. LIGHTHILL ${ }^{6}$,

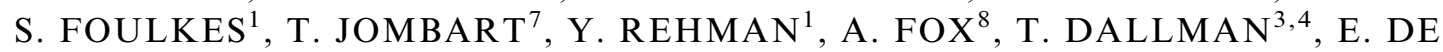 \\ PINNA $^{4}$, L. BROWNING ${ }^{9}$, J. E. COIA ${ }^{10}$, O. EDEGHERE ${ }^{1}$ AND R. VIVANCOS ${ }^{1,2,3}$ \\ ${ }^{1}$ Field Epidemiology Services, Public Health England, UK; ${ }^{2}$ University of Liverpool, UK; ${ }^{3}$ NIHR Health \\ Protection Research Unit in Gastrointestinal Infections, University of Liverpool, UK; ${ }^{4}$ Gastrointestinal \\ Bacteria Reference Unit, Public Health England, Colindale, UK; ${ }^{5}$ Instituto de Salud Carlos III, Laboratorio \\ de Referencia e Investigación en Enfermedades Bacterianas Transmitidas por Agua y Alimentos. Centro Nacional \\ de Microbiología, Madrid, Spain; ${ }^{6}$ Food Standards Agency, UK; ${ }^{7}$ Department of Infectious Disease \\ Epidemiology, Imperial College London, UK, ${ }^{8}$ Food Water and Environmental Microbiology Laboratory, Public \\ Health England, UK; ${ }^{9}$ Health Protection Scotland, Gastrointestinal Disease and Zoonoses, UK; ${ }^{10}$ Scottish \\ Microbiology Reference Laboratories, Glasgow, UK
}

Received 6 May 2016; Final revision 7 July 2016; Accepted 8 August 2016; first published online 26 October 2016

\section{SUMMARY}

Since April 2015, whole genome sequencing (WGS) has been the routine test for Salmonella identification, surveillance and outbreak investigation at the national reference laboratory in England and Wales. In May 2015, an outbreak of Salmonella Enteritidis cases was detected using WGS data and investigated. UK cases were interviewed to obtain a food history and links between suppliers were mapped to produce a food chain network for chicken eggs. The association between the food chain network and the phylogeny was explored using a network comparison approach. Food and environmental samples were taken from premises linked to cases and tested for Salmonella. Within the outbreak single nucleotide polymorphism defined cluster, 136 cases were identified in the UK and 18 in Spain. One isolate from a food containing chicken eggs was within the outbreak cluster. There was a significant association between the chicken egg food chain of UK cases and phylogeny of outbreak isolates. This is the first published Salmonella outbreak to be prospectively detected using WGS. This outbreak in the UK was linked with contemporaneous cases in Spain by WGS. We conclude that UK and Spanish cases were exposed to a common source of Salmonella-contaminated chicken eggs.

Key words: Epidemiology, outbreak, salmonellosis, traceback, whole genome sequencing.

\section{INTRODUCTION}

The number of Salmonella enterica serovar Enteritidis infections has been declining in the UK since 1997

\footnotetext{
* Author for correspondence: Mr T. Inns, Public Health England, Rail House, Lord Nelson Street, Liverpool L1 1JF, UK. (Email: thomas.inns@phe.gov.uk)

Limited aspects of this work were presented at the 11th International Meeting on Microbial Epidemiological Markers (IMMEM XI), Estoril, Portugal, 11 March 2016.
}

$[1,2]$. This decrease has been due to the successful adoption of vaccination and various other control measures in poultry production [3]. However, at a European level, chicken eggs are still the most important source of foodborne Salmonella outbreaks [4]. Chicken eggs imported into the UK from other European countries have previously been associated with multiple Salmonella outbreaks [5-7].

Since April 2015, whole genome sequencing (WGS) has been used by the national reference laboratory in 
England and Wales as the routine procedure performed on all cultures of Salmonella sp. referred by local laboratories. WGS is the primary test for identification, surveillance and outbreak investigation by the reference laboratory; all other typing methods previously employed have been significantly reduced or withdrawn $[8,9]$.

At the beginning of May 2015, an initial cluster of 29 S. Enteritidis cases was detected, and the cases were within a 5 -single nucleotide polymorphism (SNP) single linkage cluster. These cases had specimens taken in a 2-month period; this was greater than the 10 cases within this 5-SNP cluster which were recorded in the whole of 2014. Twenty-three of the 29 cases were typed as $S$. Enteritidis phage type 59 (PT59). An outbreak was declared and Public Health England (PHE) led a multi-agency outbreak control team (OCT). The objective of the OCT was to identify the source of infection and implement control measures to prevent further cases.

\section{METHODS}

\section{Case definition}

Cases were ascertained from statutory notifications of cases of Salmonella infection. In the UK, local diagnostic laboratories are required to refer all Salmonella isolates to the national reference laboratories for confirmation and characterization. Cases were defined as persons resident in Great Britain and British Overseas Territories, with an onset of illness between 1 January and 27 November 2015 , with $S$. Enteritidis infection which was whole genome sequenced and was within a specific single linkage cluster at the 10-SNP level. Cases were defined as travel associated if they had a history of travel outside the UK in the 7 days before onset of illness.

\section{WGS}

Sequencing was carried out by the PHE Genome Sequencing and Development Unit using Nextera library preparation and Illumina HiSeq 2500 (Illumina Inc., USA) in fast-run mode according to the manufacturer's instructions. High-quality Illumina reads were mapped to the $S$. enterica Enteritidis reference genome (GenBank accession no. AM933172) using BWA-MEM [10]. SNPs were then identified using GATK2 in unified genotyper mode [11]. Genome positions that had a high-quality SNP ( $>90 \%$ consensus, minimum depth $10 x, G Q \geqslant 30$ ) in at least one strain were extracted and RAxML v. 8.1.17 used to derive the maximum-likelihood phylogeny of the isolates [12]. Correlation between root to tip length and sample date was calculated using Path-O-Gen v. 1.4 (http://tree.bio.ed.ac.uk/software/ pathogen/). Hierarchical single linkage clustering was performed on the pairwise SNP difference between all isolates at various distance thresholds $(\Delta 250, \Delta 100$, $\Delta 50, \Delta 25, \Delta 10, \Delta 5, \Delta 0)$. The result of the clustering is a SNP address that can be used to describe the population structure based on clonal groups. FASTQ reads from all sequences in this study can be found at the PHE Pathogens BioProject at the National Center for Biotechnology Information (accession no. PRJNA248792), sample accession numbers are given in Supplementary Table S1. In addition to WGS, all isolates were phage-typed by the Salmonella Reference Service according to the method of Ward et al. [13].

\section{Food chain investigation}

Cases were interviewed using local questionnaires to ascertain foods eaten and other exposures in the 5 days before onset of symptoms. These questionnaires differed depending on where in the UK the case was interviewed. Shops, restaurants and other food outlets identified by cases were investigated by environmental health officers and the Food Standards Agency. Due to the previous associations between $S$. Enteritidis outbreaks and chicken egg consumption [5-7], food chain investigations concentrated on chicken eggs and chicken. From each point of sale, distributors and suppliers were traced by members of the OCT. Links between suppliers were mapped to produce a food chain network using yED Graph Editor v. 3.14 [14].

\section{Network comparison}

Potential links between the food chain network and the phylogeny were explored by comparing distances on the food chain network to genetic distances between isolates. Distances on the food chain network were measured as the number of intermediate nodes on the shortest path between pairs of cases (e.g. the distance between two cases infected at the same premises will be 1). Genetic distances between cases were computed as the patristic distances between the corresponding tips of the phylogeny. A Monte-Carlo Mantel test was used to assess the degree of correlation between the supply network and genetic distance 
matrices, using cases that were both sequenced and included in the food chain network; 9999 random permutations of the data were used to generate the null distribution of the Mantel correlation. Because such a relationship may be driven by cases infected by the same source, we also repeated this analysis using pairwise comparisons of cases with different sources of infection only. These analyses were carried out in R [15], using igraph [16] for graph distances, adephylo [17] for phylogenetic distances computations and ade4 [18] for the Mantel test.

\section{Food and environmental sampling}

When our food chain investigations identified premises associated with several cases, environmental and/or food samples were taken, where possible. Food and environmental samples including raw shell chicken eggs (both shell and contents were analysed in pools of up to 10 eggs) were tested for Salmonella using the PHE national method FNES16 [19]. All presumptive Salmonella isolates were confirmed biochemically or by PCR and referred to the PHE Salmonella Reference Service for further confirmation and typing.

\section{Spanish investigations}

Due to epidemiological information which suggested a substantial proportion of cases had a history of travel to Spain, the National Centre of Microbiology, Institute of Health Carlos III, Madrid, Spain was contacted. Phage-typing is the primary method used by the Salmonella Reference Unit in Spain to type $S$. Enteritidis isolates. In Spain, 144 S. Enteritidis PT59 isolates were recorded in 2015, compared to an average of 28 in the previous 3 years. To investigate the link to cases in the UK, a convenience sample of $19 S$. Enteritidis PT59 isolates (18 from human cases, one from a food sample) from 2015 were sent to PHE for WGS. Eleven of these isolates were from sporadic cases and eight were from two outbreaks linked to eggs; four isolates from humans in one outbreak, three human and one food isolate in the other outbreak.

\section{RESULTS}

\section{Descriptive epidemiology}

A total of 136 cases were identified, of which 21 were travel associated. Twenty-six (19·1\%) cases were hospitalized. The cases' ages ranged between 2 months and 98 years (median 31 years); 24 cases were aged
$<10$ years. Seventy-six $(56 \%)$ cases were female. The first onset of illness was on 16 February 2015; the last date of onset was on 29 September 2015, the distribution of onset dates is shown in Figure 1. There appear to be two peaks of incidence, with the first peak at week 15 , followed by a decrease and a second peak around week 35 .

The geographical distribution of cases is shown in Supplementary Figure S1. The majority $(90 \%)$ of cases were resident in England, 12 in Scotland and one in Wales. Non-travel-associated cases showed a strong degree of clustering around the centre of the UK; cases were most frequently from the West Midlands $(n=40)$ and North West $(n=33)$ regions. Of the 21 travelassociated cases, travel to Spain was reported by 12 , travel to Portugal reported by three and two had links with Gibraltar; one travelled there and one was resident there. In total, $17(81 \%)$ of the 21 travel-associated cases had a history of travel to Spain or the Iberian Peninsula in the 7 days before onset of illness.

\section{Microbiological results}

Phage-typing showed that $92(68 \%)$ of the cases corresponded to PT59. Twenty-three cases were untypable, 10 were PT14b, five were RDNC (reacts but does not conform), three were PT56, two were PT62 and one was PT8.

\section{Spanish isolates}

All 18 isolates from Spanish cases were within the outbreak specific single linkage cluster at the 10-SNP level. For the convenience sample of Spanish cases; ages ranged between 3 and 104 years (median 44), 56\% were female and specimen dates ranged between 8 April and 18 October 2015. The isolate from a food sample also fell within the outbreak-specific linkage cluster at the 10-SNP level. This food sample was from an omelette, sampled on 29 April 2015 as it was linked to three cases. It contained chicken eggs which were traced to a supermarket. Microbiological analysis of chicken eggs from the same lot was not positive for Salmonella spp. The eggs were traced to a farm in Spain which was inspected; this inspection did not indicate any infraction and microbiological investigation was negative.

\section{Phylogenetic results}

The outbreak forms a monophyletic cluster within the broader phylogeny of $S$. Enteritidis (Fig. 2). The 


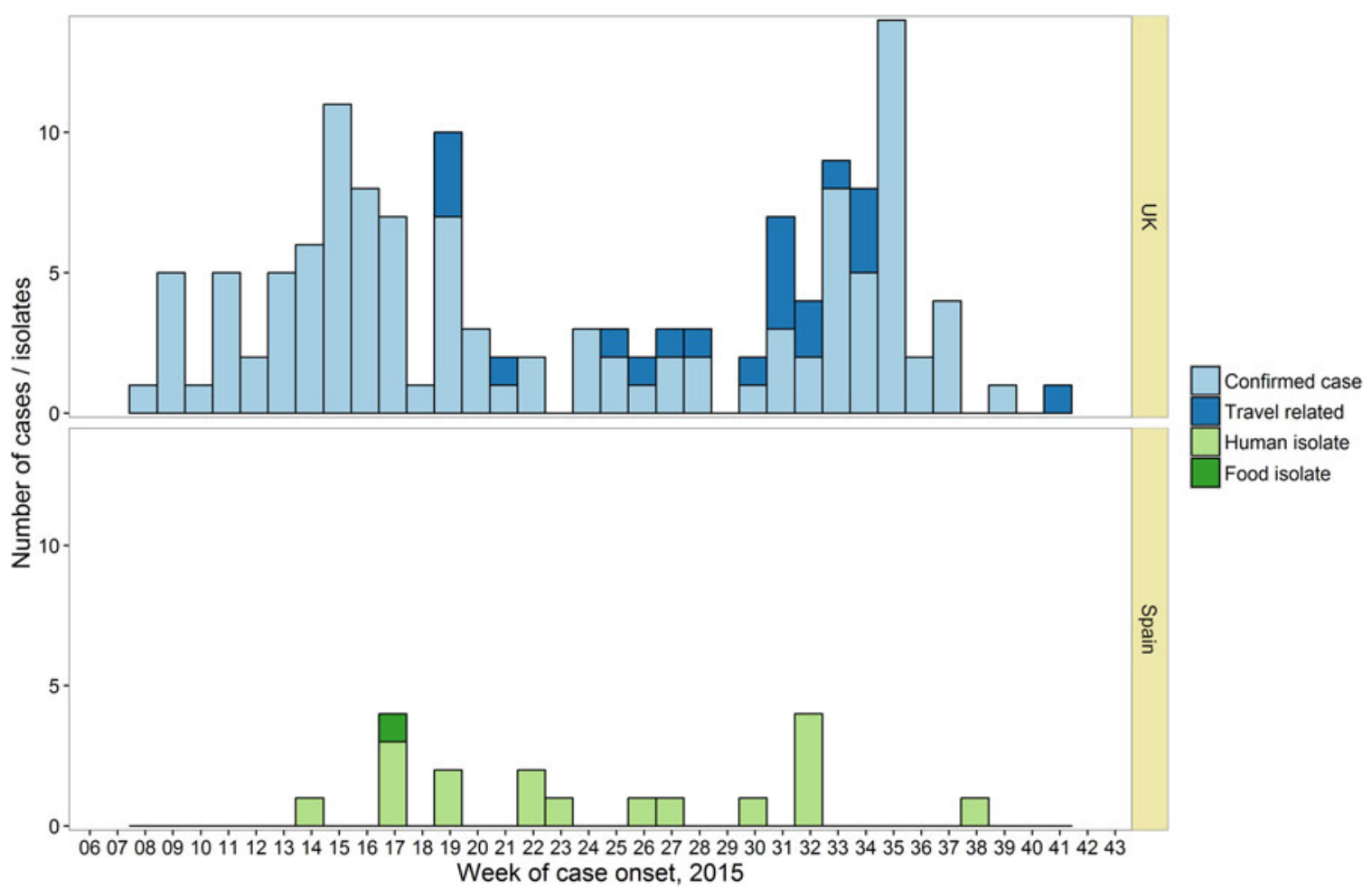

Fig. 1. Distribution of cases over time, by resident country of case. Specimen date used when onset date unavailable, 2015 $(n=40)$.

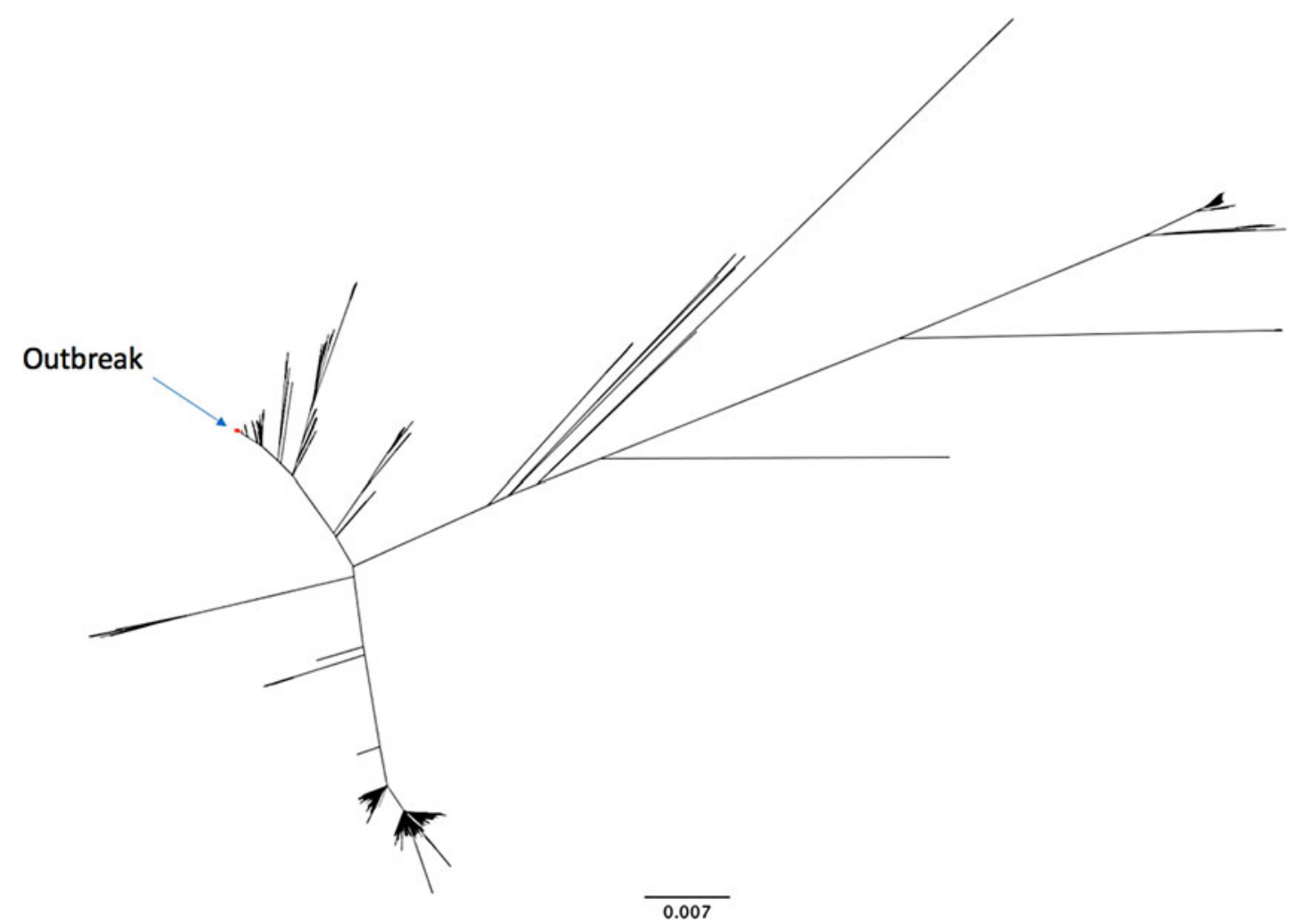

Fig. 2. An unrooted phylogenetic tree of 742 diverse Salmonella Enteritidis (eBurst group 4), built with 28117 variant positions. The 136 outbreak isolates are highlighted in red. 
Inner column - outbreak time period (week)

Outer column - is the isolate linked to Spain?
08 to 22

23 to 29

301039

Yes

No

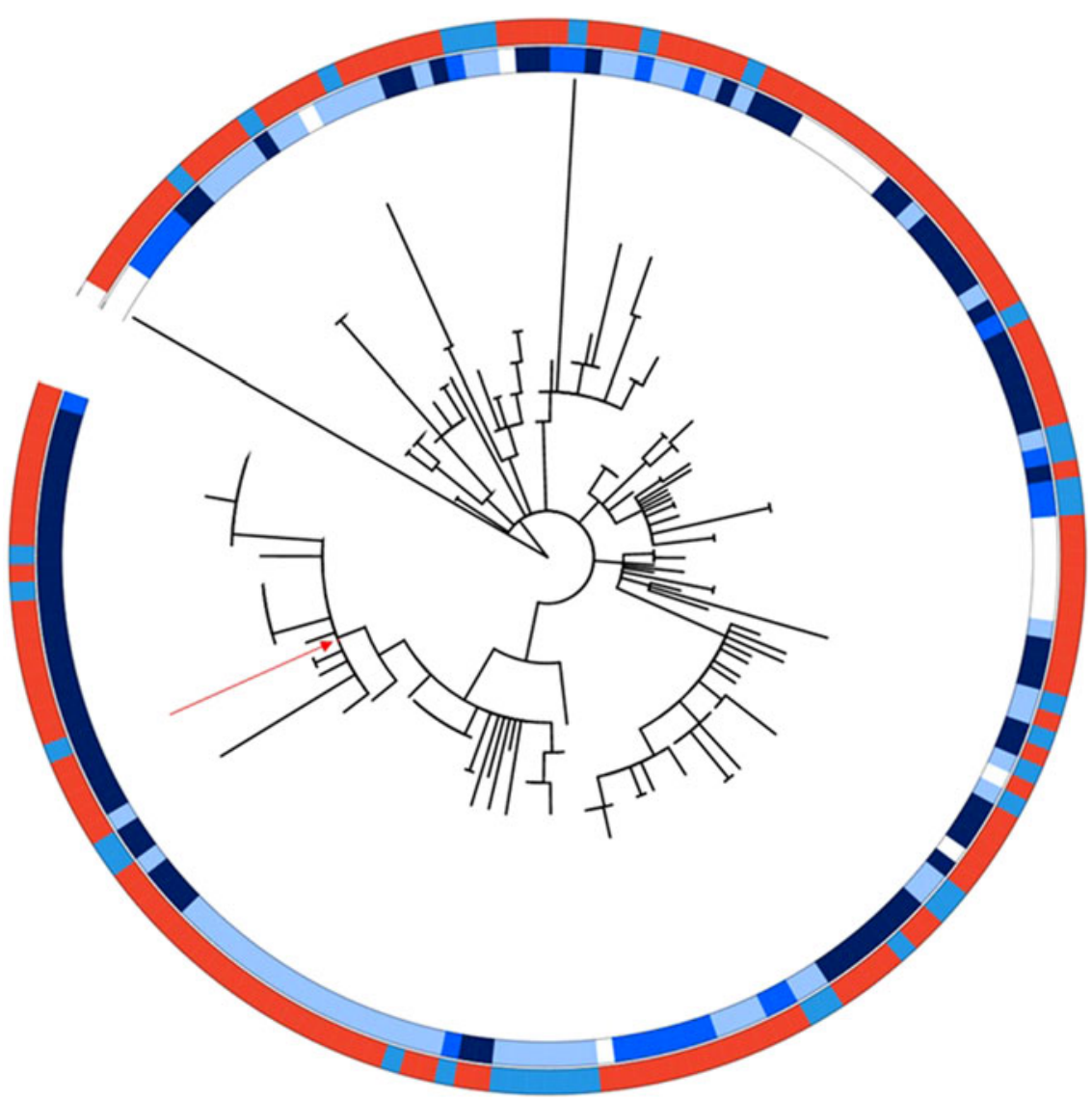

Fig. 3. Maximum likelihood phylogenetic tree of the whole genome sequencing cluster containing the outbreak strains. Food isolate node label is highlighted in red.

nearest strain to the outbreak cluster (apart from previous cases, detailed below) was 11 SNPs away. The median pairwise distance between isolates meeting the outbreak definition was 10 SNPs (s.D. $=3.9$ SNPs), with a maximum SNP distance of 20 SNPs (Supplementary Fig. S2); the phylogenetic tree (Fig. 3) also reflects this diversity. Within the five clusters where $\geqslant 3$ cases reported exposure at the same food establishment, the median pairwise SNP distance was $0 \mathrm{SNPs}$, with a maximum of $2 \mathrm{SNPs}$. Isolates associated with the Iberian Peninsula by residence or travel clustered throughout the tree. There was weak correlation between root to tip distance of the phylogeny and date of isolation (correlation coefficient $=0 \cdot 53$, Supplementary Fig. S3).

\section{Food chain investigation}

Food exposure information was available for $87(63 \%)$ cases. Of these, $63(72 \%)$ had a history of eating out in the 5 days before onset. These cases visited 41 premises; chicken egg supply information could be obtained for 31 of these premises; 23 premises could be linked to a chicken egg supply network. The chicken egg supply network produced by this investigation is shown in Figure 4. Of the 87 cases with food 


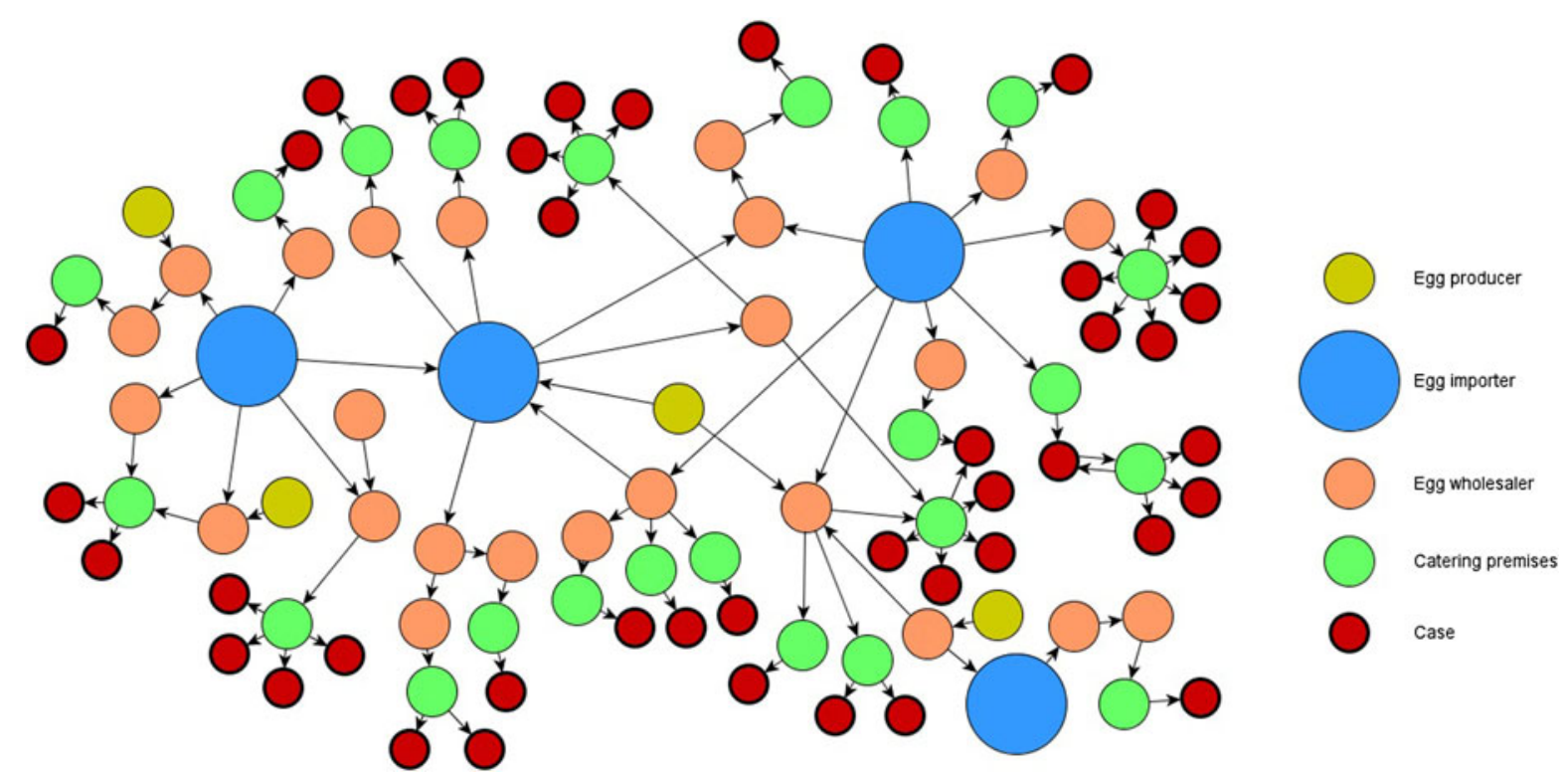

Fig. 4. Summary of egg food chain network, arrows show inferred direction of egg supply or exposure (total cases $=43$ ).

exposure information, $43(49 \%)$ cases could be plausibly linked to this network.

\section{Food and environmental sampling}

A total of 17 catering premises, egg wholesalers and importers were sampled by environmental health officers from local authorities. A total of 1962 chicken eggs were sampled; none were positive for Salmonella sp. Twenty-six environmental samples were taken from catering premises; none were positive for Salmonella sp. Twelve chicken samples were taken; two raw chicken samples were positive for $S$. Livingstone and $S$. Enteritidis, respectively. The $S$. Enteritidis isolated from the chicken was $>100$ SNPs distant from the outbreak cluster, although by phagetyping the isolates were RDNC, which would not have allowed it to be ruled out as a causative agent without further typing. Six samples of other foods were taken; none were positive for Salmonella sp.

\section{Network comparison}

According to the Monte-Carlo Mantel test, genetic distances between isolates significantly increased with the distance (number of intermediate nodes) on the distribution network $(r=0 \cdot 32, P=0 \cdot 0001)$ (Fig. 5). This relationship remained significant, albeit substantially weaker, when considering only pairs of cases from different origins $(r=0 \cdot 07, t$ test: $P=0 \cdot 03749)$, and was also robust to nonlinearities between distances (Spearman's $\rho=$ $0.08, P=0.02676$ ).

\section{Previous cases}

Sequences from isolates in this outbreak were uploaded to the GenomeTrakr database hosted at the National Center for Biotechnology Information (NCBI) [20]. An initial K-mer analysis was used to screen for similar isolates; these were then downloaded from the GenomeTrakr database and subjected to SNP analysis as described in the Methods section. SNP analysis showed that three cases from New York State were within the outbreak-specific single linkage cluster at the 10-SNP level. Follow-up information was only available for two of these cases. Both cases had onset of illness in 2014 and had a history of travel to Europe during their incubation period; one case visited Paris and one visited both Paris and London. In addition, there were 17 cases in residents of the UK with an onset of illness in 2014 and with a Salmonella isolate which was within the outbreak-specific single linkage cluster at the 10-SNP level (Fig. 3).

\section{DISCUSSION}

Here we describe an outbreak of $S$. Enteritidis in the UK that was linked to contemporaneous cases in Spain; these cases were within a specific single linkage cluster at the 10-SNP level. We found that cases were 


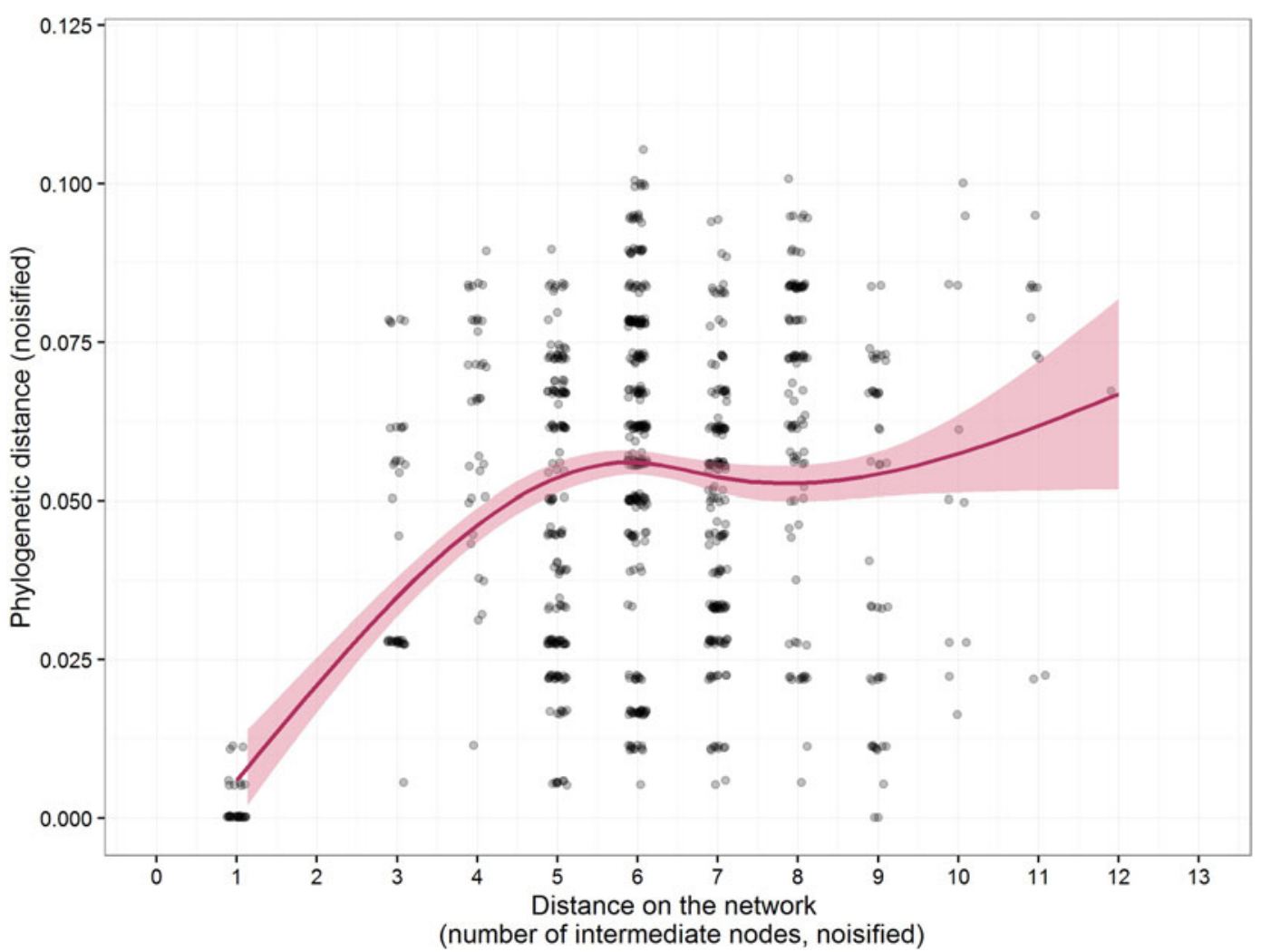

Fig. 5. Jitter plot showing the relationship between the phylogenetic distance and the distance between cases on the food chain network, with cubic spline.

associated with chicken eggs; a food isolate within the outbreak cluster was taken in Spain from an omelette linked to several Salmonella cases. In addition, findings from food chain analysis for UK cases suggest that a number of these cases were linked by a chicken egg supply network; this network was significantly correlated with the phylogeny of the isolates. From this, we infer that UK and Spanish cases were exposed to a common source of Salmonella-contaminated chicken eggs.

This is the first published Salmonella outbreak to be prospectively detected using WGS. One of the main benefits of using WGS as the primary test for identification, surveillance and outbreak investigation is that it provides additional sensitivity over phage-typing (the previous routine typing technique for $S$. Enteritidis and a few other serovars) and far greater specificity in linking to isolates from other laboratories. Of the UK cases, 68\% corresponded to PT59. Had WGS not been used, it is likely that the outbreak would still have been recognized, as phage-typing surveillance has previously been effective in detecting outbreaks [5]. The loss of $32 \%$ of cases would have likely slowed the recognition of the outbreak, and made it harder to pull together a food chain network, although whether the same source would have been identified is a matter of speculation.

An increasing number of countries are using WGS of Salmonella routinely for surveillance and outbreak detection [21, 22]. Due to information sharing and the use of databases such as GenomeTrakr, the ability to investigate multi-country Salmonella infections by comparing sequence information is greatly enhanced. Routine WGS also changes the way an outbreak is managed; it was previously accepted practice in infectious intestinal disease outbreaks to exclude cases with travel history to focus on possible in-country exposures. This practice does not preclude the inclusion of cases with foreign travel if the suspect source is from or in another country, but often the origin of the suspect source is not known. With the greater specificity of WGS information, travel histories and other geographical metadata can now provide information on which other countries may have cases from the same source [23].

$S$. Enteritidis is considered a monomorphic pathogen that is hard to discriminate with previous typing methods (e.g. MLVA or PFGE) [24, 25]. Therefore, 
there may be an expectation that Enteritidis outbreaks analysed by WGS would form clusters with no detectable changes in their genomes. However, here we present an epidemiologically and genomically linked outbreak with considerable diversity, with a median SNP pairwise distance of 10 SNPs. We hypothesize that this is due to the outbreak being sampled from bacterial populations with a large effective population size, possibly due to contamination of multiple farms from the same source. The fact that isolates of this SNP cluster were also seen in 2014 is consistent with the source being chicken eggs, as laying hens can have a working life of 20 months ( 35 with moulting) [26], and Salmonella contamination could conceivably persist in a flock over this entire period, or be transferred between consecutive flocks without detection [27-29]. We show that older isolates tended to be closer to the root of the tree, which is consistent with sampling from a persistent population over time. WGS also allowed the linking of three cases from 2014 from the United States as well, two of which were epidemiologically linked with Europe. This finding was facilitated by the GenomeTrakr project and New York State Department of Health colleagues, and is an example of the remarkable sensitivity and specificity of WGS. This type of international collaboration and comparison of WGS information provides exciting opportunities to enhance the safety of our global food supply [30].

Despite the hypothesized association between these cases and chicken eggs, one of the limitations of this investigation is that we did not isolate Salmonella from any of the eggs sampled during this outbreak investigation. It has been previously documented that the time between chicken egg consumption and chicken egg sampling is typically greater than the shelf life of chicken eggs [5]. In this investigation we sampled 1962 chicken eggs and none were positive for Salmonella. The Spanish Reference Laboratory investigated $48 \mathrm{~S}$. Enteritidis isolates from laying hens isolated during the salmonellosis control and prevention programme in 2015. PT59 was not detected in any of those isolates. It was, however, possible to isolate Salmonella in the outbreak cluster from an omelette in Spain, and there was a significant association between the chicken egg food chain and the genome phylogeny.

Food chain information was difficult to obtain in this outbreak; food exposure information was only available for $63 \%$ of cases; of 41 premises identified, egg supply information was obtained for only 31 .
This situation arose both from the challenges in contacting cases and poor recall, but also the very resource-intensive nature of traceback investigations to identify the food chain. In the European Union, food business operators (including importers) are legally required to ensure that on investigation, traceability can be assured at all stages (EC Regulation 178/2002). The experience of investigators in this outbreak was that this information was frequently unavailable and often very time-consuming to obtain.

Comparisons between the food chain network and phylogeny produced from WGS provide a promising new analytical application of this method for outbreak investigation [31]. Regarding the food chain information used in this analysis, it is important to remember that although local questionnaires asked similar questions, there could be variation between authorities in the questionnaires used and resources available for interview. If this difference in collecting information systematically varied across areas or countries, this could potentially affect the extent of the food chain network and any inferences drawn from comparisons using it.

In this outbreak, we have shown the way in which the routine use of WGS for Salmonella surveillance has provided additional opportunities for outbreak detection and investigation. The ability to collaborate on an international level in a timely and resource-efficient fashion would be enhanced by the use of an international database with good European and worldwide coverage. Ideally, this would include coverage of samples taken for farm and food monitoring, and for veterinary purposes. The greater use of WGS has also provided opportunities to develop new analytical methods to compare phylogenies with other sources of information for outbreak investigations. To make the most of these new methods, food chain investigations are of increasing importance, we recommend that national authorities work with food businesses to ensure they can meet legal requirements for traceability.

\section{SUPPLEMENTARY MATERIAL}

For supplementary material accompanying this paper visit http: //dx.doi.org/10.1017/S0950268816001941.

\section{ACKNOWLEDGEMENTS}

This paper is written on behalf of the Outbreak Control Team; we acknowledge the contribution of 
all members, in particular Debbie Anderson for administrative support. We also acknowledge the work of environmental health officers in obtaining both information for the food chain investigation and food and environmental samples. We also thank Lesley Larkin and Rob Davies for advice on Salmonella in the poultry industry.

Thanks are also due to Bill Wolfgang and Madhu Anand from the New York State Department of Health for depositing their sequencing data and sharing their follow-up information. We also thank the GenomeTrakr initiative for organizing the data in such a way that it is possible to find closely related cases for further SNP analysis.

T.J. is funded by the Medical Research Council Centre for Outbreak Analysis and Modelling and the National Institute for Health Research-Health Protection Research Unit for Modelling Methodology. T.I., T.D. and R.V. are supported by the National Institute for Health Research Health Protection Research Unit (NIHR HPRU) in Gastrointestinal Infections in partnership with Public Health England (PHE). The views expressed are those of the authors and not necessarily those of the NHS, the NIHR, the Department of Health or PHE.

\section{DECLARATION OF INTEREST}

None.

\section{REFERENCES}

1. Lane CR, et al. Salmonella enterica serovar Enteritidis, England and Wales, 1945-2011. Emerging Infectious Diseases 2014; 20: 1097-104.

2. O'Brien SJ. The 'decline and fall' of nontyphoidal Salmonella in the United Kingdom. Clinical Infectious Diseases 2013; 56: 705-710.

3. Hugas M, Beloeil P. Controlling Salmonella along the food chain in the European Union - progress over the last ten years. Eurosurveillance 2014; 19: 20804.

4. EFSA and ECDC (European Food Safety Authority and European Centre for Disease Prevention and Control). The European Union summary report on trends and sources of zoonoses, zoonotic agents and food-borne outbreaks in 2013. EFSA Journal 2015; 13: 3991.

5. Inns T, et al. A multi-country Salmonella Enteritidis phage type $14 \mathrm{~b}$ outbreak associated with eggs from a German producer: 'near real-time' application of whole genome sequencing and food chain investigations, United Kingdom, May to September 2014. Eurosurveillance 2015; 20: 21098.

6. Janmohamed K, et al. National outbreak of Salmonella Enteritidis phage type $14 \mathrm{~b}$ in England, September to
December 2009: case-control study. Eurosurveillance 2011; 16.

7. Harker KS, et al. National outbreaks of Salmonella infection in the UK, 2000-2011. Epidemiology and Infection 2014; 142: 601-607.

8. Ashton PM, et al. Identification and typing of Salmonella for public health surveillance using whole genome sequencing. PeerJ 2015; 4: e1752.

9. Ashton PM, et al. Revolutionising public health reference microbiology using whole genome sequencing: Salmonella as an exemplar. bioRxiv preprint 2015. doi: http://dx.doi.org/10.1101/033225.

10. Li H, Durbin R. Fast and accurate long-read alignment with Burrows-Wheeler transform. Bioinformatics 2010; 26: $589-595$.

11. McKenna A, et al. The Genome Analysis Toolkit: a MapReduce framework for analyzing next-generation DNA sequencing data. Genome Research 2010; 20: 1297-1303.

12. Stamatakis A. RAxML version 8: a tool for phylogenetic analysis and post-analysis of large phylogenies. Bioinformatics 2014; 30: 1312-1313.

13. Ward LR, De Sa JDH, Rowe B. A phage-typing scheme for Salmonella Enteritidis. Epidemiology and Infection 1987; 99: 291-294.

14. yED Graph Editor. Version 3.14 (http: //www.yworks. com/en/products/yfiles/).

15. R Core Team. R: A language and environment for statistical computing. R Foundation for Statistical Computing, Vienna, Austria.

16. Csardi G, Nepusz T. The igraph software package for complex network research. InterJournal Complex Systems 2006, 1695.

17. Jombart T, Dray S. adephylo: exploratory analyses for the phylogenetic comparative method. Bioinformatics 2010; 26: 1907-1909.

18. Dray S, Dufour AB. The ade4 package: implementing the duality diagram for ecologists. Journal of Statistical Software 2007; 22: 1-20.

19. Public Health England. Detection of Salmonella species. Microbiology Services. Food, Water \& Environmental Microbiology Standard Method FNES16 (F13); version 2, 2014 (https://www.gov.uk/government/uploads/ system/uploads/attachment_data/file/330682/National_ SOP_FNES16_F13_Detection_of_Salmonella_Species. pdf). Accessed 7 April 2016.

20. U.S. Food and Drug Administration. Whole Genome Sequencing (WGS) Program (http://www.fda.gov/Food/ FoodScienceResearch/WholeGenomeSequencingProgram WGS/default.htm). Accessed 25 February 2016.

21. den Bakker HC, et al. Rapid whole-genome sequencing for surveillance of Salmonella enterica serovar Enteritidis. Emerging Infectious Diseases 2014; 20: 1306-1314.

22. Leekitcharoenphon $\mathbf{P}$, et al. Evaluation of whole genome sequencing for outbreak detection of Salmonella enterica. PLOS ONE 2014; 9: e87991.

23. Hoffmann M, et al. Tracing origins of the Salmonella Bareilly strain causing a food-borne outbreak in the United States. Journal of Infectious Diseases 2016; 213: $502-508$. 
24. Zheng J, et al. Enhanced subtyping scheme for Salmonella Enteritidis. Emerging Infectious Diseases 2007; 13: 1932-1935.

25. Boxrud D, et al. Comparison of multiple-locus variable-number tandem repeat analysis, pulsed-field gel electrophoresis, and phage typing for subtype analysis of Salmonella enterica serotype Enteritidis. Journal of Clinical Microbiology 2007; 45: 536-543.

26. Yousaf M, Chaudhry A. History, changing scenarios and future strategies to induce moulting in laying hens. World's Poultry Science Journal 2008; 64: 65-75.

27. Carrique-Mas JJ, et al. Persistence and clearance of different Salmonella serovars in buildings housing laying hens. Epidemiology and Infection 2009; 137: 837-846.

28. Arnold ME, Carrique-Mas JJ, Davies RH. Sensitivity of environmental sampling methods for detecting
Salmonella Enteritidis in commercial laying flocks relative to the within-flock prevalence. Epidemiology and Infection 2010; 138; 330-339.

29. Davies RH, Wales AD. Developments in Salmonella control in eggs. In: Sofos J, ed. Advances in Microbial Food Safety. Oxford, UK: Woodhead Publishing, 2015, pp. 281-311.

30. Dunn JR. Whole-genome sequencing: opportunities and challenges for public health, food-borne outbreak investigations, and the global food supply. Journal of Infectious Diseases 2016; 213: 499-501.

31. Dallman T, Inns T, Jombart T, et al. Phylogenetic structure of European Salmonella Enteritidis outbreak correlates with national and international egg distribution network. Microbial Genomics. Published online 26 May 2016. doi:10.1099/mgen.0.000070. 